\title{
SMALL INTESTINE
}

\section{An immunodominant DQ8 restricted gliadin peptide activates small intestinal immune response in in vitro cultured mucosa from HLA-DQ8 positive but not HLA-DQ8 negative coeliac patients}

\author{
G Mazzarella, M Maglio, F Paparo, G Nardone, R Stefanile, L Greco, Y van de Wal, \\ Y Kooy, F Koning, S Auricchio, R Troncone
}

See end of article for authors' affiliations

Correspondence to:

Professor R Troncone,

Dipartimento di Pediatria

Università Federico II, via

Sergio Pansini 5, 1-80131

Napoli, Italy;

troncone@unina.it

Accepted for publication 11 July 2002

\begin{abstract}
Background: Studies on intestinal T cell clones from the mucosa of patients with coeliac disease have led to the identification of immunogenic gliadin epitopes. One is HLA-DQ8 restricted, its recognition by $T$ cells being increased by introduction of negatively charged residues operated by tissue transglutaminase.

Aim: To test HLA-DQ8 restricted epitope in both native (QYPSGQGSFQPSQQNPQA) and deamidated (QYPSGEGSFQPSQENPQA) forms in an organ culture system of treated coeliac mucosa from HLA-DQ8 positive and HLA-DQ8 negative patients.

Patients and methods: Jejunal biopsies obtained from 10 patients with coeliac disease (six HLA-DQ8 positive and four HLA-DQ8 negative) were cultured in vitro with a peptic-tryptic digest (PT) of gliadin, or with the native (peptide A) or deamidated (peptide B) peptide. Intraepithelial $\mathrm{CD}^{+}$and lamina propria total $\mathrm{CD}_{25} 5^{+}$and $\mathrm{CD} 3^{+} \mathrm{CD} 25^{+}$cells were counted, lamina propria intercellular adhesion molecule 1 (ICAM-1) expression was evaluated, as well as that of Fas molecules on epithelial cells.

Results: In HLA-DQ8 positive, but not in HLA-DQ8 negative, coeliacs the density of intraepithelial CD3 ${ }^{+}$ cells, lamina propria total $C D 25^{+}$, and $C D 3^{+} C D 25^{+}$cells, as well as expression of ICAM- 1 and Fas molecules were significantly increased in biopsies cultured with PT, peptide A, or peptide B compared with biopsies cultured in medium alone.

Conclusion: These data show that the DQ8 restricted gliadin peptide is immunogenic only in the intestinal mucosa of HLA-DQ8 positive coeliac patients in both native and deamidated forms.
\end{abstract}

Abbreviations: $C D$, coeliac disease; $L P M N C$, lamina propria mononuclear cells; PT, peptic-tryptic digest; TBS, Tris buffered saline; PAP, peroxidase-antiperoxidase; APAAP, alkaline phosphatase/antialkaline phosphatase; PBS, phosphate buffered saline; ICAM-1, intercellular adhesion molecule 1. 
Table 1 HLA DQ molecular typing of subjects investigated in this study

\begin{tabular}{lllll}
\hline Patient No & DQA ${ }^{*}$ & \multicolumn{3}{c}{ DQB ${ }^{*}$} \\
\hline \multicolumn{2}{l}{ HLADQ8 positive subjects } \\
1 & 0301 & 0301 & 0302 & 0302 \\
2 & 0301 & 0301 & 0302 & 0302 \\
3 & 0501 & 0301 & 0201 & 0302 \\
4 & 0501 & 0301 & 0201 & 0302 \\
5 & 0301 & 0301 & 0302 & 0302 \\
6 & 0501 & 0301 & 0201 & 0302 \\
HLADQ8 & negative subjects & & & \\
7 & 0501 & 0501 & 0201 & 0201 \\
8 & 0501 & 0101 & 0201 & 0503 \\
9 & 0501 & 0301 & 0201 & 0301 \\
10 & 0501 & 03 & 03 & 0201 \\
\hline
\end{tabular}

extraction method. Amplification of the polymorphic second exon of DRB1, DQAl, and DQBl genes, and dot blot analysis of amplified DNA with sequence specific oligonucleotides probes was carried out. HLA typing of patients is reported in table 1. Six patients were DQ8 positive but three were also DQ2 positive. Four patients were DQ2 positive, all being DQ8 negative. Here, the first six will be referred to as DQ8 positive and the remaining four as DQ8 negative.

\section{Peptides}

A peptic-tryptic digest (PT) of gliadin (variety S Pastore, gift of Dr De Vincenzi, Istituto Superiore di Sanita', Rome, Italy) was used as a positive control. Peptide A corresponds to the residues 203-220 (sequence QYPSGQGSFQPSQQNPQA of gda09, SwissProt P18573). Its deamidated form (peptide B) presents two residues of glutamine converted to glutamic acid in positions 208 and 216 (QYPSGEGSFQPSQENPQA). Peptides were synthesised as previously reported.'

Processing of jejunal biopsies and in vitro organ culture Four biopsy specimens from the proximal jejunum were obtained from each patient, and all specimens were placed immediately in ice chilled $0.15 \mathrm{~mol} / \mathrm{l}$ sodium chloride and brought to the laboratory within 30 minutes. One specimen from each patient was appropriately oriented and embedded in OCT compound (Tissue Tek, Miles Laboratories, Elkhart, Indiana, USA), snap frozen in isopenthane, cooled in liquid nitrogen, and stored at $-70^{\circ} \mathrm{C}$ until cryosectioning. The remaining pieces were placed on a stainless steel mesh positioned over the central well of an organ culture dish (Becton Dickinson, New York, USA) with the mucosal surface of the biopsies uppermost. Culture took place as previously reported $^{13}$ in the presence of media alone, PT gliadin digest ( $1 \mathrm{mg} / \mathrm{ml}$ ), or the native (peptide A $1 \mathrm{mg} / \mathrm{ml}$ ) or deamidated (peptide B $1 \mathrm{mg} / \mathrm{ml}$ ) peptide. The dishes were placed in a sterile anaerobic jar which was gassed with 95\% oxygen $/ 5 \%$ carbon dioxide and incubated at $37^{\circ} \mathrm{C}$. After 24 hours of culture, the tissue was embedded in OCT compound, snap frozen in liquid nitrogen, and prepared for cryosectioning.

\section{Staining technique}

Cryostat sections $(5 \mu \mathrm{m})$ were air dried at room temperature and fixed in acetone for 10 minutes. All sections were repeatedly washed at room temperature in Tris buffered saline (TBS), incubated in normal rabbit serum (1:100 in TBS for 30 minutes), and then stained according to the peroxidaseantiperoxidase (PAP) or alkaline phosphatase/antialkaline phosphatase (APAAP) method. They were individually tested with the monoclonal antibodies (table 2). After one hour of incubation at room temperature, sections were incubated for 30 minutes with rabbit antimouse serum (Dako, Milan, Italy) and for a further 30 minutes with APAAP complex to stain CD25 positive cells and intercellular adhesion molecule 1 (ICAM-1) positive cells, or with PAP complex (Dako) to stain CD3 and CD95 (Fas) positive cells. Slides were washed in TBS for 10 minutes following each antibody incubation and were developed by a final incubation of five minutes with 2 amino-9 ethyl-carbazole (Sigma) when the PAP complex was used; when the APAAP complex was used instead, the immune reaction product was developed by stirring continuously for three minutes with $20 \mathrm{mg}$ of naphthol-AS-biphosphate in 0.5 $\mathrm{ml} N$ - $N$-dimethylformamide added to $0.2 \mathrm{ml}$ of sodium nitrite (Sigma Chemical Company, St Louis, Missouri, USA ), $0.08 \mathrm{ml}$ of New Fucsin (Merck, West Point, Pennsylvania, USA), $40 \mathrm{ml}$ of TBS, pH 8.7, and $17.5 \mathrm{mg}$ of Levamisole (Sigma). Omission of the primary antibody served to control against non-specific antibody binding. The sections were finally stained with Mayer's haematoxylin and mounted. Non-immune mouse immunoglobulins at the same isotype were used as the primary antibody for control of specificity. In experiments to detect activated $\mathrm{T}$ cells $\left(\mathrm{CD}^{+}{ }^{+} \mathrm{CD} 25^{+}\right)$, immunofluorescence with confocal microscopy was used because it allows better resolution of three colour procedures. Crysections were fixed in acetone and incubated for one hour at room temperature with a mixture of mouse monoclonal antibodies anti-CD25 human (Dako, 1:25) and rabbit polyclonal anti-CD3 human (Dako, l:100), followed by a mixture of horse antimouse FITC coniugated ( $1: 200$, Vector Laboratories, California, USA) and swine antirabbit TRITC coniugated (Dako, 1:300). These antibody were applied for 45 minutes in the dark followed by incubation (30 minutes) with ToPro-3 (Molecular Probes Leiden, the Netherlands) for counterstaining the nuclei. Finally, sections were mounted in phosphate buffered saline (PBS):glycerol (1:1). The primary and secondary antibodies were diluted appropriately in PBS containing bovine serum albumin at $1 \%$, and all incubations took place at room temperature with intervening 10 minute PBS rinses.

$\mathrm{CD}^{+}$and $\mathrm{CD}_{2} 5^{+}$immunofluorescence cells were imaged with a Leica SP confocal microscope (Germany) using a 40× Plan-Neofluar oil immersion objective. The illumination source was from three single lasers: $488 \mathrm{~nm}$ line from argon ion laser, $543 \mathrm{~nm}$ line from HeNe ion laser, and $633 \mathrm{~nm}$ line from HeNe ion laser.

\section{Morphometric analysis}

The density of cells expressing CD3 in the intraepithelial compartment was determined by counting the number of stained cells as a percentage of 100 enterocytes; the number of CD25

\begin{tabular}{lllll}
\hline \multicolumn{4}{l}{ Table 2} & \multicolumn{4}{l}{ Monoclonal and polyclonal antibodies used in this study } \\
\hline Designation & Specificity & Isotype & Working dilution & Source \\
\hline T3-4B5 & CD3 & $\operatorname{lgG} 1$ & $1: 200$ & Dako \\
ACT-1 & CD25 & $\lg 1$ & $1: 25$ & Dako \\
B-2 & ICAM-1 & $\lg 1$ & $1: 10$ & Becton Dickinson \\
DX2 & CD95 & $\lg 1$ & $1: 100$ & Pharmingen \\
T-cell & CD3 1 & Rabbit lgG & $1: 50$ & Dako \\
\hline
\end{tabular}




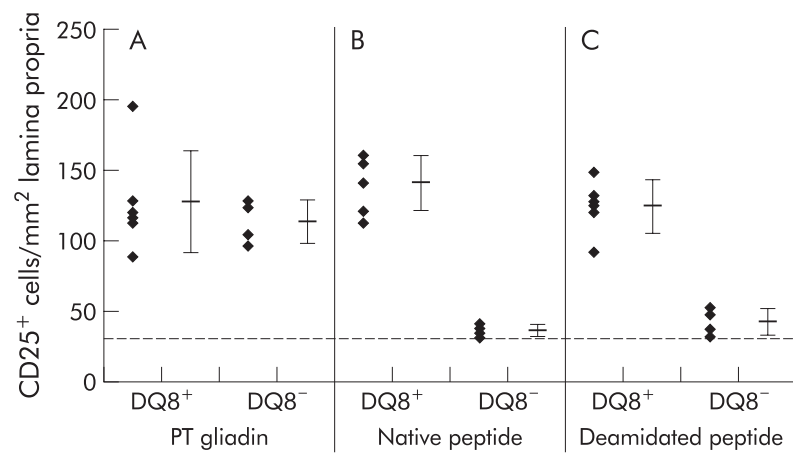

Figure 1 CD25 mononuclear cells in the lamina propria of jejunal biopsy specimens from HLA-DQ8 ${ }^{+}$and HLA-DQ8 ${ }^{-}$coeliac patients cultured in vitro with peptic-tryptic digest (PT) of gliadin, native peptide (peptide A), or deamidated peptide (peptide B). The broken line represents values obtained in fragment cultured with medium alone. Values are means (SD).

Table 3 Percentage of $\mathrm{CD}^{+}$lamina propria lymphocytes expressing CD25 after culture with medium alone, peptic-tryptic digest of gliadin, or native or deamidated peptide

\begin{tabular}{lll}
\hline & DQ8 $^{+}$patients & DQ8 $^{-}$patients \\
\hline Medium & $1.7(0.8)^{*}$ & $1.8(0.7)$ \\
Peptic-triptic digest of gliadin & $9.1(3.3)$ & $9.3(1.4)$ \\
Native peptide & $6.3(1.4)$ & $2.4(0.8)$ \\
Deamidated peptide & $7.1(1.6)$ & $2.5(0.9)$ \\
\hline *Data are mean (SD). & & \\
\end{tabular}

lamina propria mononuclear cells (LPMNC) was evaluated within a total area of $1 \mathrm{~mm}^{2}$ of lamina propria using a microscope with a calibrated ocular aligned parallel to the muscularis mucosae. Lamina propria $\mathrm{CD}^{+} \mathrm{CD} 25^{+}$cells were counted as a percentage of the total $\mathrm{CD}^{+}$cells; at least 500 $\mathrm{CD}^{+}$cells were evaluated in each sample. Staining of epithelial cells by anti-CD95 and LPMNC by anti-ICAM-1 was evaluated in terms of staining intensity and graded on an arbitrary scale of weak staining $(+)=1$, moderate staining $(++)=2$, or strong staining $(+++)=3$. Counts were independently analysed in a blinded manner by two observers.

\section{Statistical analysis}

The Student's two tailed $t$ test was used to compare specimens exposed to gliadin or synthetic peptides with those exposed to medium alone for the same incubation time. Non-parametric tests (Wilcoxon two tailed) were also applied and the results are concordant with those obtained using parametric tests.

\section{RESULTS}

Effects of in vitro HLA-DQ8 restricted peptide challenge on $\mathrm{T}$ cell activation

For both HLA-DQ8 ${ }^{+}$and HLA-DQ8 ${ }^{-}$coeliac patients, in jejunal biopsies cultured in the presence of a PT digest of gliadin, the number of cells $/ \mathrm{mm}^{2}$ expressing the interleukin 2 receptor (CD25 cells) (mean (SD) 121 (40) and 113 (15), respectively) was significantly $(\mathrm{p}<0.001)$ higher than in those cultured in medium alone (28 (6) and 26 (8), respectively) (fig 1). However, only in HLA-DQ8 ${ }^{+}$coeliacs was the number of CD25 $5^{+}$ cells in specimens cultured in the presence of native peptide $(139(22))$ or deamidated peptide $(124(21))$ significantly higher $(\mathrm{p}<0.001)$ than in those cultured in medium alone. In fact, no significant differences were noted in HLA-DQ8 ${ }^{-}$ coeliac patients between biopsy specimens cultured with native peptide $(37(2))$ or deamidated peptide (38 (10)) and those cultured in only medium (26 (8)) (fig 1). In further
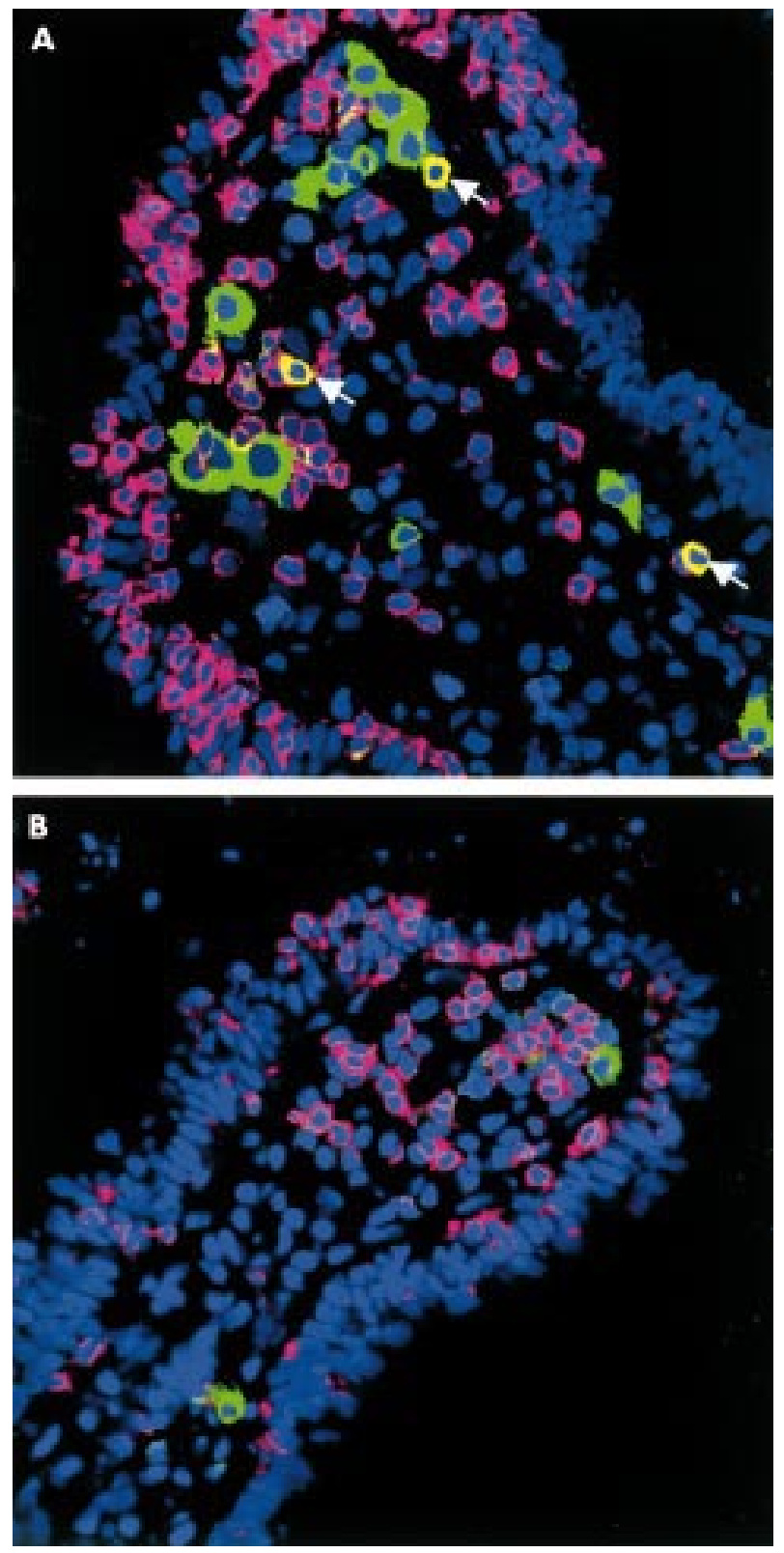

Figure 2 Immunofluorescence staining of jejunal mucosa from a $D Q 8^{+}$coeliac patient cultured in vitro with deamidated peptide $(A)$ or with medium only (B). (A) Increase in $\mathrm{CD}^{+}$intraepithelial lymphocytes (red) is evident compared with (B); also evident is the increase in $\mathrm{CD} 25^{+}$cells (green), particularly in the subepithelial region. A significant increase in $\mathrm{CD} 3^{+} \mathrm{CD} 25^{+}$activated T cells (yellow) was also noted. A similar pattern was observed when the mucosa was cultured with the native peptide or with the peptic-tryptic digest of gliadin.

experiments, we examined the increase in $\mathrm{CD}^{+} \mathrm{CD} 25^{+}$cells; in fact, both HLA-DQ8 ${ }^{+}$and HLA-DQ8 ${ }^{-}$coeliac patients challenged in vitro with the PT digest of gliadin showed a significant increase $(p<0.01)$ in activated T cells; nevertheless, only HLA-DQ8 ${ }^{+}$coeliac patients showed a significant increase $(\mathrm{p}<0.05)$ of $\mathrm{CD}^{+} \mathrm{CD} 25^{+}$cells in response to native or deamidated peptide (table 3 , fig 2 ).

Expression of ICAM-1 was also evaluated to find further evidence of activated mucosal cell mediated immunity. ICAM-1 was increased significantly in all cells and vessels of biopsy specimens from HLA-DQ8 ${ }^{+}(\mathrm{p}<0.001)$ and HLA-DQ8 $(\mathrm{p}<0.01)$ coeliac patients cultured for 24 hours with PT gliadin compared with those cultured with medium alone; in contrast, only in coeliac HLA-DQ8 ${ }^{+}$was ICAM-1 expression 


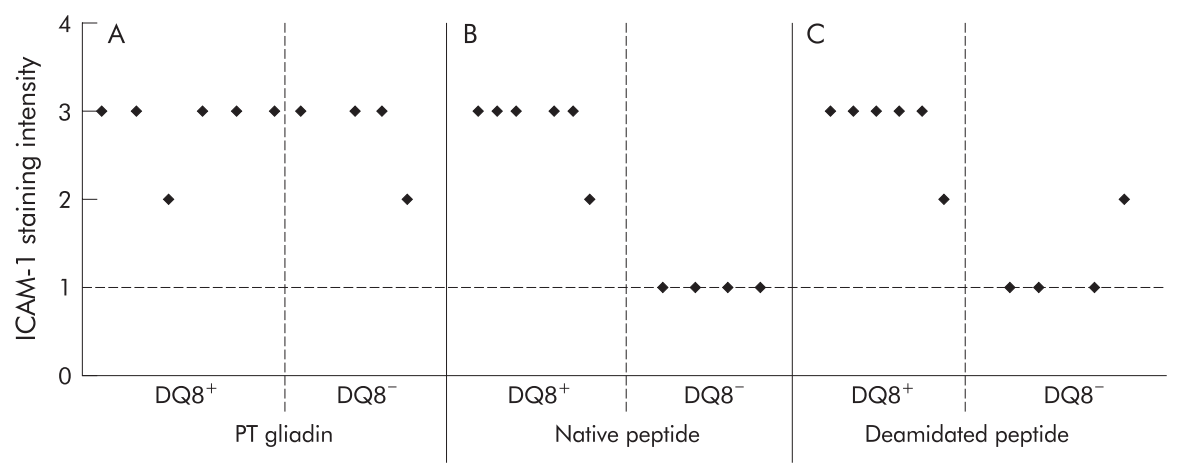

Figure 3 Intercellular adhesion molecule-1 (ICAM-1) expression in the lamina propria of jejunal biopsy specimens from HLA-DQ8 ${ }^{+}$and HLA-DQ8 $8^{-}$coeliac patients cultured in vitro with peptic-tryptic digest (PT) of gliadin, native peptide (peptide A), or deamidated peptide (peptide B). Expression of adhesion molecules was evaluated in terms of staining intensity and graded on an arbitrary scale of weak staining $(+)=1$, moderate staining $(++)=2$, and strong staining $(+++)=3$. The broken line represents values obtained in fragment cultured with medium alone.

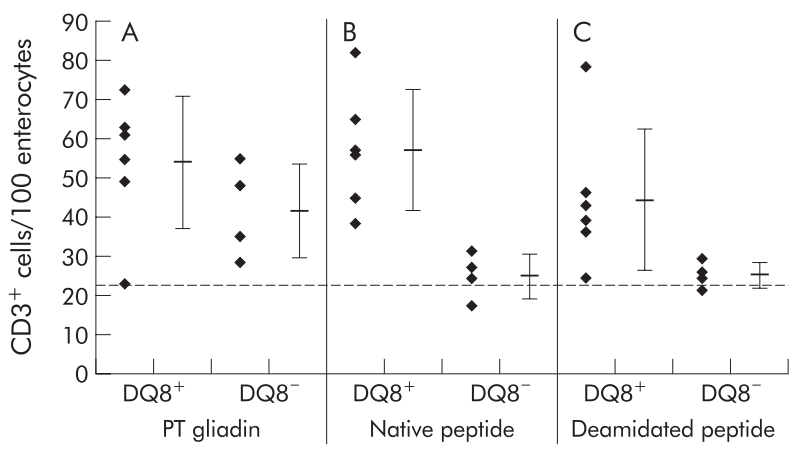

Figure 4 CD3 intraepithelial lymphocytes in jejunal biopsy specimens from HLA-DQ8 ${ }^{+}$and HLA-DQ8 ${ }^{-}$coeliac patients cultured in vitro with peptic-tryptic digest (PT) of gliadin, native peptide (peptide A), or deamidated peptide (peptide B). The broken line represents values obtained in fragment cultured with medium alone. Values are means (SD).

significantly enhanced in the presence of native peptide or deamidated peptide $(\mathrm{p}<0.001)$ while no significant differences were noted when ICAM-1 expression was evaluated in biopsies of HLA-DQ8 $8^{-}$patients cultured in the presence of the peptides (both native and deamidated) in comparison with culture with medium alone (fig 3).

Effects of in vitro HLA-DQ8 restricted peptide challenge on $\mathrm{T}$ cell infiltration

A significant increase in CD3 intraepithelial lymphocytes was seen in HLA-DQ8 ${ }^{+}$biopsies cultured in the presence of the PT digest of gliadin $(54(19))(\mathrm{p}<0.001)$, native peptide (54 (19)) $(\mathrm{p}<0.001)$, or deamidated peptide $(44 \quad(20)) \quad(\mathrm{p}<0.01)$ compared with those cultured in medium alone (23 (9)) (fig 4 , fig 2). In HLA-DQ8 ${ }^{-}$coeliac patients, a significant $(\mathrm{p}<0.01)$ increase in $\mathrm{CD} 3$ intraepithelial lymphocytes was seen only in biopsy specimens cultured in the presence of gliadin (42 (12)). In fact, no significant differences were noted in the number of $\mathrm{CD}^{+}$intraepithelial lymphocytes when biopsies of HLA-DQ8 patients were cultured in the presence of the DQ8 restricted peptides (both native (26 (8) and deamidated (25 (4)) compared with those cultured in medium alone (21 (8)) (fig $4)$.

\section{Effects of in vitro HLA-DQ8 restricted peptide challenge} on Fas epithelial expression

Epithelial expression of Fas, particulary evident in basolateral membranes of enterocytes, was increased after 24 hours of challenge with PT gliadin, both in biopsy samples obtained from HLA-DQ8 ${ }^{+}(\mathrm{p}<0.001)$ and HLA-DQ8 ${ }^{-}(\mathrm{p}<0.01)$ coeliac patients compared with samples cultured in the presence of medium alone (fig 5). Also, significantly enhanced expression was seen when HLA-DQ8 ${ }^{+}$coeliac biopsies were cultured in the presence of native peptide $(\mathrm{p}<0.001)$ or deamidated peptide $(\mathrm{p}<0.001)$ compared with those cultured in medium alone (figs 5, 6). This phenomena was specific for HLA-DQ8 ${ }^{+}$ coeliac patients; in fact, no significant differences were noted when Fas expression was evaluated in biopsies of HLA-DQ8 patients cultured in the presence of the DQ8 restricted peptides (both native and deamidated).

\section{DISCUSSION}

Most evidence suggests that a decisive role in the pathogenesis of coeliac disease is played by HLA-DQ2 and/or DQ8 restricted

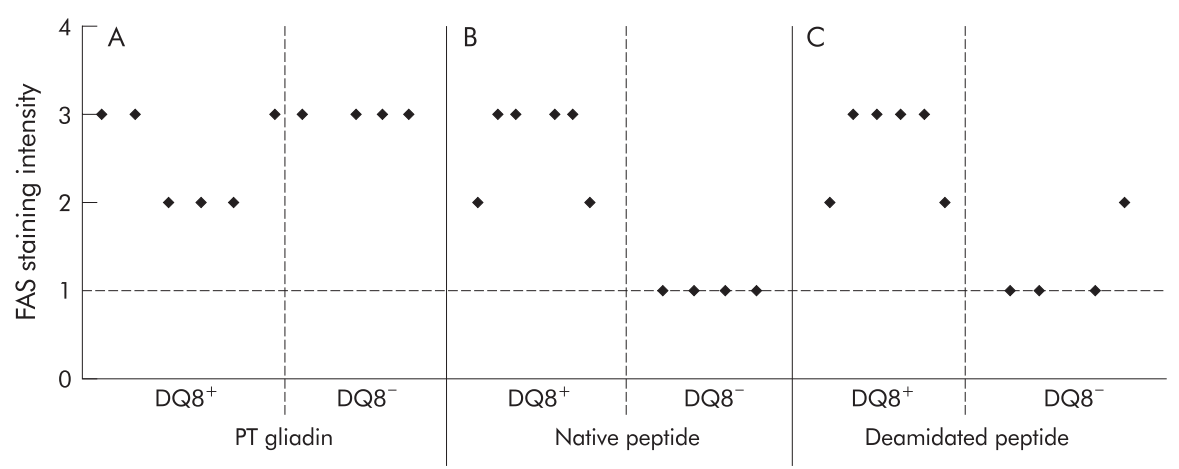

Figure 5 Epithelial cell Fas expression in the jejunal mucosa from HLA-DQ8 $8^{+}$and HLA-DQ8 ${ }^{-}$coeliac patients cultured in vitro with peptic-tryptic digest (PT) of gliadin, native peptide (peptide A), or deamidated peptide (peptide B). Expression of Fas was evaluated in terms of staining intensity and graded on an arbitrary scale of weak staining $(+)=1$, moderate staining $(++)=2$, and strong staining $(+++)=3$. The broken line represents values obtained in fragment cultured with medium. 

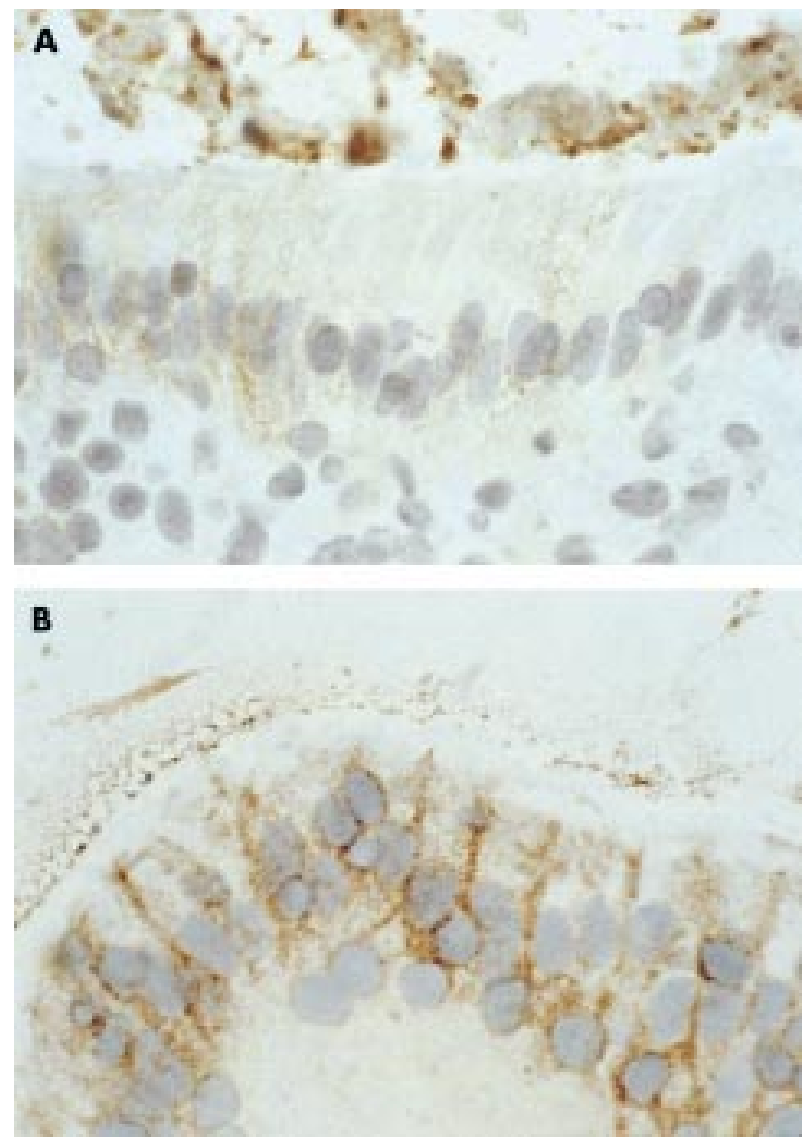

Figure 6 Fas expression in the epithelium of jejunal mucosa from a $D Q 8+$ coeliac patient cultured in vitro with medium only $(A)$ or with native peptide (peptide A) (B). In the latter, intense staining is detected in almost all of the epithelial cells; expression is particulary evident in basolateral membranes of enterocytes. A similar pattern was observed when the mucosa was cultured with the deamidated peptide or with the peptic-tryptic digest of gliadin. Original magnification $\times 100$

gluten specific T cells at the site of the lesion in the gut. ${ }^{17}$ None the less, because of the considerable biochemical complexity of gluten proteins, there is still uncertainty about the amino acid sequences which are responsible for mucosal damage. Several tools have been used over the years to identify "toxic" sequences, probably investigating different properties of gliadin peptides not always related to T cell recognition. ${ }^{14}{ }^{15}$ Most recently, considerable progress in the identification of $\mathrm{T}$ cell stimulatory gluten derived peptides has been rendered possible by the isolation of gliadin specific $\mathrm{T}$ cell clones from the mucosa of treated and untreated patients, and by the implementation of modern analytical tools. This work has proved that deamidation can enhance $\mathrm{T}$ cell reactivity to such peptides. To date, five unique epitopes of gliadin that are recognised by $\mathrm{T}$ cells have been identified, three restricted by HLA-DQ2 and two by HLA-DQ8 molecules, but many more are predicted to be immunogenic. ${ }^{16}$ Reactivity of all HLA-DQ2 restricted epitopes and one of the HLA-DQ8 restricted peptides is significantly enhanced by deamidation of specific glutamine residues to glutamic acid. ${ }^{4-9}$ This does not seem to be true for the remaining HLA-DQ8 determinant which belongs to a glutenin molecule. ${ }^{17}$

We aimed to transfer these observations to a more complex system represented by the in vitro organ culture of coeliac biopsies, a model already used in the past to test the biological properties of gliadin peptides ${ }^{14}$ and, more recently, their immunogenicity. ${ }^{10}{ }^{13}$ Our data showed that the DQ8 restricted gliadin epitope, identified for its activity on T cell clones, ${ }^{89}$ was also active in the organ culture system of the treated coeliac mucosa. In this system, it significantly increased the density of lamina propria mononuclear cells and in particular $\mathrm{T}$ cells expressing the interleukin 2 receptor. The extent of the latter phenomenon, which is accompanied by a redistribution of these cells, recruited in the subepithelial compartment, is of similar amplitude to that observed in cultures performed in the presence of the whole gliadin PT digest. The most likely explanation is that the peptide used is certainly immunodominant but it is also possible that activation of T cell clones specific for that peptide is followed by a bystander activation of other mononuclear cells; in fact, in our culture system, following gliadin stimulation, the dominating $\mathrm{CD} 25^{+}$subset was represented by $\mathrm{CD} 3-\mathrm{CD} 25^{+}$cells, the latter being presumably activated macrophages.

We have also observed significant enhancement of expression of ICAM-1 in those fragments cultured in the presence of the peptide. The latter phenomenon has already been described in treated coeliac biopsies cultured with a gliadin digest ${ }^{18}$ and is attributed to the strong $\gamma$-interferon induction which is one of the main features of this in vitro response. ${ }^{19}$ Moreover, two other phenomena have been observed in our biopsies cultured with the peptide: increased intraepithelial density of $\mathrm{CD}^{+}$cells and enhanced expression of Fas molecules on epithelial cells. The first of such observations is a phenomenon already shown to occur in vitro in response to gliadin; as it was not affected by the presence of CTLA4-Ig, a molecule interfering with costimulation of $\mathrm{T}$ cells, its $\mathrm{T}$ cell dependence has been questioned, and a pathway parallel to that involving mucosal T cells has been hypothesised.$^{18}$ Similar observations have been made for induction of Fas for which a direct effect on epithelial cells (non-T cell mediated) has been suggested. ${ }^{18}$ Yet, it is still possible that CD3 epithelial infiltration is a consequence of lamina propria $\mathrm{T}$ cell activation. In fact, in explants of human fetal gut, small intestine T cell activation by anti-CD3 antibodies induced a significant increase in intraepithelial lymphocytes, suggesting that it might be a consequence of lamina propria $\mathrm{T}$ cell activation. ${ }^{20}$ More difficult to explain is Fas expression on epithelial cells as there is no previous evidence suggesting the influence of $\mathrm{T}$ cell activation on such phenomenon, which in our experience seems to be DQ restricted. The relatively large amount of peptide used in the system must also be considered in interpreting the results.

None of these phenomena was observed in HLA-DQ8 negative subjects. This clear HLA-DQ restriction of peptide activity has strong implications not only for our understanding of the sequence of events triggered by gliadin in the coeliac mucosa; in fact, for the first time gliadin peptides have been shown to display different biological activities in different subsets of coeliac patients. It is still unclear if the response of coeliac patients is directed against a few or multiple peptides, but our observations raise the issue of their immunogenicity, depending on the genetic make up of the patient. For the peptide that we investigated, it is likely to be immmunodominant as all six HLA-DQ8 coeliac mucosae tested showed a clear response.

No differences were noted between the native or deamidated form of the peptide when all in vitro responses were analysed. Both peptides may be active, even if to different extents, as suggested by $\mathrm{T}$ cell clones studies. The relatively large amount used in the organ culture system could allow even the non-deamidated peptide to activate T cells; in this case, deamidation would not be crucial. In the alternative hypothesis, only the deamidated peptide is active; as deamidation is likely to take place during organ culture, ${ }^{21}$ fragments cultured with the non-deamidated peptide would eventually become activated. Experiments aimed at blocking in situ tissue transglutaminase activity could help to establish the possible role of mucosal deamidation of the native peptide.

In conclusion, we have evidence that a peptide identified and characterised for its activity on $\mathrm{T}$ cell clones isolated from 
coeliac mucosae is also active, in a HLA-DQ restricted manner, in an in vitro organ culture system, causing T cell activation, intraepithelial migration of T cells, and preapoptotic phenomena. The organ culture system is confirmed as very useful in determining the biological activity of gliadin peptides, before the ultimate proof of their toxicity is provided by challenge studies.

\section{ACKNOWLEDGEMENTS}

This study was carried out with financial support from the Commission of the European Communities, specific RTD programme "Quality of Life and Management of Living Resources", QLK1-CT-1999-00037, Evaluation of the prevalence of coeliac disease and its genetic components in the European population. This work was partly supported by the "Istituto Superiore di Sanita': Sorveglianza degli effetti nocivi da alimenti: intolleranza ai cereali (Convenzione 98/JK/T3)". The technical help of Mr C Meccariello and Dr L Cipriano (Institute of Food Science and Technology, CNR Avellino, Italy) is gratefully acknowledged.

\section{Authors' affiliations}

G Mazzarella, R Stefanile, Institute of Food Science and Technology, CNR Avellino, Italy

M Maglio, F Paparo, L Greco, S Auricchio, Department of Paediatrics and European Laboratory for the Investigation of Food-Induced Diseases, University Federico II, Naples, Italy

G Nardone, Department of Experimental Medicine, University Federico II, Naples, Italy

Y van de Wal, Y Kooy, F Koning, Department of Immunohaematology and Blood Transfusion, University Medical Centre, Leiden, the Netherlands

R Troncone, Institute of Food Science and Technology, CNR Avellino, and Department of Paediatrics and European Laboratory for the Investigation of Food-Induced Diseases, University Federico II, Naples, Italy

\section{REFERENCES}

1 Auricchio S, Troncone R, Maurano F. Coeliac disease in the year 2000 Ital J Gastroenterol Hepatol 1999:31:773-80.

2 Nilsen EM, Brandtzaeg P. Mucosal cytokine expression in coeliac disease. In: Auricchio S, Greco L, Maiuri L, et al, eds. Coeliac disease. Naples: JGC Editions, 1999:75-8.

3 Kagnoff MF. HLA genes in coeliac disease In: Auricchio S, Greco L, Maiuri L, et al, eds. Coeliac disease. Naples: JGC Editions, 1999:5-14

4 Sjostrom $\mathrm{H}$, Lundin KEA, Molberg $\mathrm{O}$, et al. Identification of a gliadin T-cell epitope in coeliac disease: general importance of gliadin deamidation for intestinal T-cell recognition. Scand J Immunol 1998:48:111-15.
5 Molberg O, McAdam SN, Korner R, et al. Tissue transglutaminase selectively modifies gliadin peptides that are recognized by gut-derived $T$ cell. Nat Med 1998;4:713-17.

6 Arentz-Hansen $\mathbf{H}$, Korner $\mathrm{R}$, Molberg $\mathrm{O}$, et al. The intestinal T cell response to alpha-gliadin in adult celiac disease is focused on a single deamidated glutamine targeted by tissue transglutaminase. J Exp Med 2000;191:603-12.

7 Sollid LM. Molecular basis of coeliac disease. Ann Rev Immunol 2000;18:53-81.

8 Van de Wal Y, Kooy YM, Van Veelen PA, et al. Small intestinal T cells of celiac disease patients recognize a natural pepsin fragment of gliadin. Proc Natl Acad Sci USA 1998;95:10050-4.

9 Van de Wal Y, Kooy YM, Van Veelen PA, et al. Selective deamidation by tissue tranglutaminase strongly enhances gliadin-specific $T$ cell reactivity. J Immunol 1998;161:1585-8.

10 Maiuri $L$, Picarelli A, Boirivant $M$, et al. Definition of initial immunologic modifications upon in vitro gliadin challenge in the small intestine of celiac patients. Gastroenterology 1996;1 10:1368-78.

11 Maiuri L, Troncone R, Mayer M, et al. In vitro activities of A-gliadin-related synthetic peptides: damaging effect on the atrophic coeliac mucosa and activation of mucosal immune response in the treated coeliac mucosa. Scand J Gastroenterol 1996;31:247-53.

12 Congia $M$, Cucca $F$, Frau $F$, et al. A gene dosage effect of the DQA 1*0501/DQB 1*0201 allelic combination influences the clinical heterogeneity of celiac disease. Hum Immunol 1994;40: 138-42.

13 Fais S, Maiuri L, Pallone F, et al. Gliadin induced changes in the expression of MHC-class II antigens by human small intestinal epithelium. Organ culture studies with coeliac disease mucosa. Gut 1992;33:472-5.

14 Silano $M$, De Vincenzi $M$. Bioactive antinutritional peptides derived from cereal prolamines: a review. Nahrung 1999;43:175-84.

15 McAdam SN, Sollid LM. Getting to grips with gluten. Gut 2000:47:743-5.

16 Vader LW, de Ru A, van Der Wal Y, et al. Specificity of tissue transglutaminase explains cereal toxicity in celiac disease. J Exp Med 2002; 195:643-9.

17 Van de Wal Y, Kooy Y, Van Veelen PA, et al. Glutenin is involved in the gluten-driven mucosal T cell respose. Eur J Immunol 1999;29:3133-9.

18 Maiuri L, Auricchio S, Coletta S, et al. Blockage of T-cell costimulation inhibits T- cell action in celiac disease. Gastroenterology 1998; 115:564-72.

19 Nilsen EM, Jahnsen FL, Lundin KEA, et al. Gluten induces an intestinal cytokine response strongly dominated by interferon gamma in patients with celiac disease. Gastroenterology 1998;115:551-63.

20 Monk T, Spencer JO, Cerf-Bensussan N, et al. Stimulation of mucosal T cells in situ with anti-CD3 antibody: location of the activated T cells and their distribution within the mucosal micro-environment. Clin Exp Immunol 1988;74:216-22.

21 Molberg O, McAdam S, Lundin KE, et al. T cells from celiac disease lesions recognize gliadin epitopes deamidated in situ by endogenous tissue transglutaminase. Eur J Immunol 2001;31:1317-23. 